\title{
«Der Dschungel hat sich in den letzten Jahren zu einer Naturwiese entwickelt»
}

\author{
Es war eine Pionierleistung: Vor 15 Jahren rief Silva Keberle das «ErfahrungsMedi- \\ zinische Register» (EMR) ins Leben mit dem Ziel, zum Schutz der Patienten Trans- \\ parenz im «Dschungel» der erfahrungsmedizinischen Angebote zu schaffen. Ein \\ Jubiläumsinterview.
}

Interview: Bruno Kesseli

bkesseli[at]emh.ch

\begin{abstract}
Frau Keberle, Sie haben vor 15 Jahren das ErfahrungsMedizinische Register (EMR) gegründet. Was hat Sie dazu motiviert?

Silva Keberle: Es waren vor allem Neugierde und Interesse an diesem weitläufigen, umstrittenen und spannenden Gebiet. Und das Bedürfnis, in diesem komplexen und damals völlig unregulierten Segment der therapeutischen Angebote den Schutz der Patienten aufzubauen. Natürlich hat das Vertrauen der Krankenversicherer in unsere Kompetenz auch eine Rolle gespielt.
\end{abstract}

habe ich selbst ausprobiert und erstaunliche, gute Erfahrungen gemacht. Bei anderen Methoden habe ich etwas Zweifel, ob die Begründung für die Wirksamkeit einer objektiven Prüfung standhalten würde. Aber ich sehe auch, dass die Schulmedizin es in den letzten Jahren etwas verpasst hat, ihre ganzheitlichen Fähigkeiten einzusetzen. Kranke Menschen brauchen - neben einer guten medizinischen Versorgung - auch viel Zuwendung, Zeit, Aufmerksamkeit. Das kommt in der doch sehr technisierten Medizin manchmal zu kurz.

\section{«Einzelne Methoden habe ich selbst ausprobiert und erstaunliche, gute Erfahrungen gemacht. Bei anderen Methoden habe ich etwas Zweifel ...»}

Welche Beziehung haben Sie selbst zur «Erfahrungsmedizin»?

Das hängt etwas von den Methoden ab. Über alle rund 120 Methoden, für die das EMR ein Qualitätslabel vergibt, könnte man meine Beziehung als neutral-interessiert bezeichnen. Einzelne Methoden

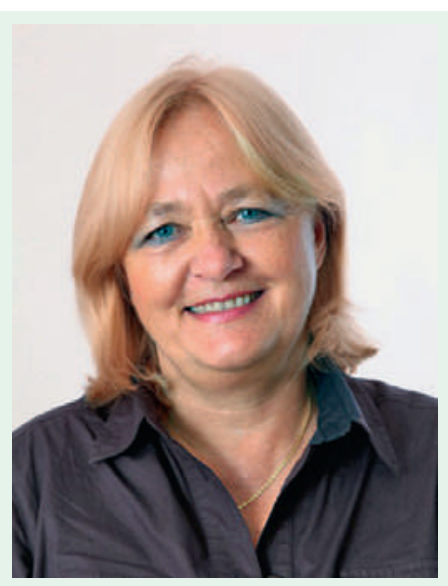

Vermutlich ist das EMR trotz seines mittlerweile 15-jährigen Bestehens vielen Ärztinnen und Ärzten noch kein Begriff. Was ist die wichtigste Funktion des EMR?

Das EMR vergibt ein Qualitätslabel für Therapeutinnen und Therapeuten der Erfahrungsmedizin, auch Komplementär- oder Alternativmedizin genannt. Mit diesem Label können die Therapeuten respektive ihre Patienten eine Behandlung zulasten der Privatversicherungen abrechnen, sofern sie eine Versicherung für Komplementärmedizin abgeschlossen haben.

Können Sie die wichtigsten Entwicklungsschritte des EMR in den vergangenen 15 Jahren kurz skizzieren?

Das sind im Wesentlichen drei grosse Schritte: zuerst die Pionierphase, als wir innert Wochen eine funktionsfähige Infrastruktur aufbauen mussten, und das in einer Zeit, als Computer und Internet noch nicht etabliert waren. Man konnte keine Begriffe «googeln», die Recherchen waren aufwendig und schwierig. So dauerte es damals zum Beispiel eine ganze Weile, bis wir herausgefunden hatten, dass die Methode «Sumathu» keine asiatische Therapieform 
ist, sondern eine Entwicklung von Herrn Sulser Max in Thun. Ausserdem mussten wir damals erst das Vertrauen der Therapeuten, der Schulen und der Verbände gewinnen.

\section{«Meines Wissens gibt es auf der ganzen Welt kein Land, das sich in diesem Gebiet so weit entwickelt hat.»}

Wie sahen die weiteren Schritte aus?

Nach einigen Jahren kam dann die Phase der Konsolidierung. Das EMR hatte sich etabliert, war enorm gewachsen und zum Marktführer geworden. Immer mehr Versicherer schlossen mit dem EMR einen Vertrag ab und nutzten unser Qualitätslabel für ihre Angebote in der Erfahrungsmedizin. enormen Sprung nach vorn gemacht. Die laufenden und kommenden Entwicklungen vorherzusehen und mit unserem Qualitätslabel diesen sich rasch wandelnden Markt abzubilden, das war und ist unsere Aufgabe in der dritten Phase.

Wenn Sie das EMR der Gründungszeit mit dem heutigen Register vergleichen: Wo liegen die grössten Unterschiede? In der Qualität der Aus- und Fortbildung der Therapeutinnen und Therapeuten. Damals konnte sich fast jeder ungehindert «Therapeut» nennen. Es gab wenige seriöse Ausbildungen und kaum klare Definitionen und Standards. Durch das EMR-Reglement wurden die ersten gesamtschweizerischen Vorgaben erarbeitet, gemeinsam mit Exponenten der Erfahrungsmedizin - das war ein Meilenstein, nicht nur in der Schweiz. Meines Wissens gibt es auf der ganzen Welt kein Land, das sich in diesem Gebiet so weit entwickelt hat.

\section{«Insgesamt ist die Qualität der Ausbildungen in den letzten 15 Jahren sehr viel besser geworden.»}

Der dritte Schritt ist die Entwicklung seit der Volksabstimmung 2009. Die Komplementärmedizin wurde in die Verfassung aufgenommen. Seitdem hat die Akzeptanz der Komplementärmedizin einen

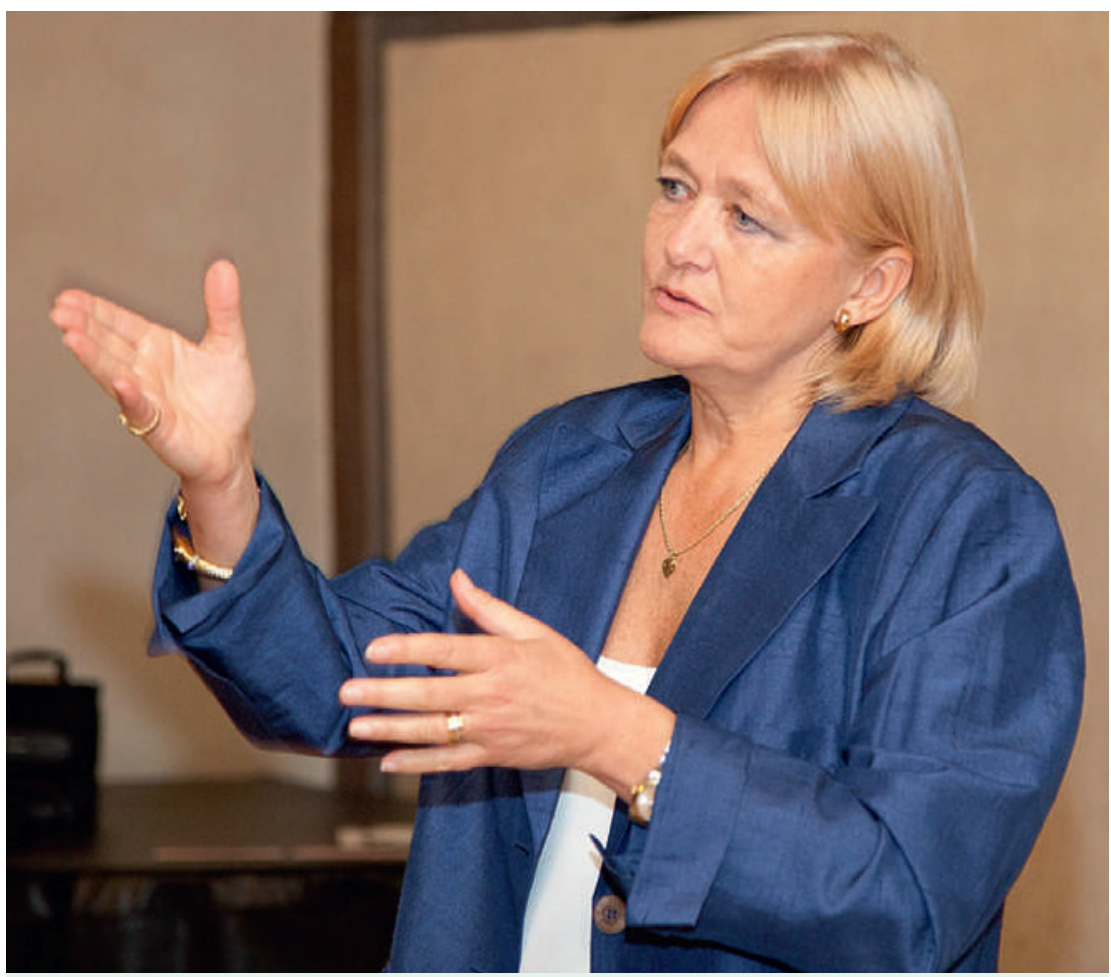

«Kaum noch abenteuerliche oder absurde Registrierungsgesuche», sagt Silva Keberle zu den Fortschritten in den vergangenen 15 Jahren.
Hat die Verankerung der Komplementärmedizin in der Verfassung nach der Abstimmung im Jahr 2009 für das EMR wesentliche Änderungen gebracht?

Ja, eindeutig. Das Thema Qualität in der Komplementärmedizin ist nun in aller Munde, zum Beispiel in der Forschung oder der Berufsbildung. Die Akzeptanz des EMR ist enorm gestiegen, das EMR ist zum Kompetenzzentrum geworden. Wir beraten nicht nur unsere Versicherer, sondern auch Kantone und andere Institutionen. Denn das EMR verfügt über eine riesige Datensammlung und kennt den Markt der Erfahrungsmedizin in der Schweiz wie keine andere Institution. Kurzum: Wir müssen weniger Überzeugungsarbeit leisten und kommen viel rascher vorwärts.

Lässt sich Qualität in der Komplementärmedizin überhaupt definieren?

Aber sicher! Qualität heisst: gute berufliche Kompetenz. Eine gute Therapeutin hat eine abgeschlossene, seriöse Ausbildung, sie bildet sich regelmässig fort, sie kennt ihre Grenzen und hält sich an die gesetzlichen Vorgaben. Dazu berücksichtigt sie einen Berufskodex, in dem der Schutz des Patienten im Vordergrund steht. All diese Kriterien finden sich im EMR-Reglement, einzusehen auf der EMR-Website.

Kann das EMR für qualitative Mindeststandards der registrierten Therapeuten Gewähr bieten?

So gut wie die FMH für ihre Ärzte Gewähr bieten kann. Was die Therapeuten in der Praxis machen, können wir nicht kontrollieren. Aber wir stellen 


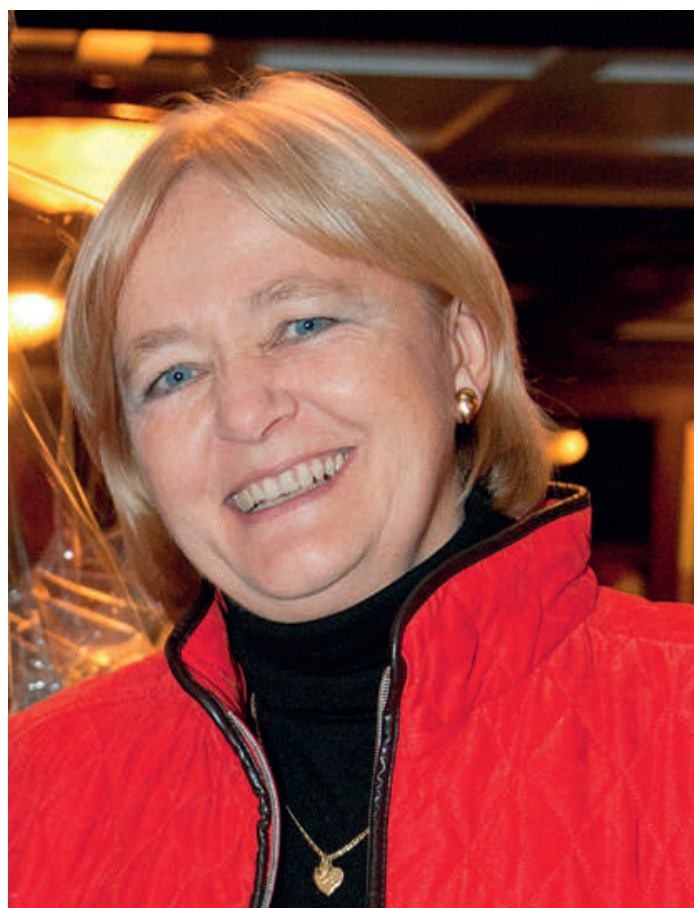

Mehr Zeit für «Aussenpolitik»: Silva Keberle hat noch einige «Eisen im Feuer».

sicher, dass die Therapeuten, die beim EMR registriert sind, sämtliche Forderungen unseres Reglements erfüllen - und das sind wie gesagt beachtlich viele! Das EMR-Reglement ist sehr umfassend. In manchen Kantonen praktizieren immer noch Personen mit kantonaler Bewilligung, die den EMR-Kriterien nicht genügen würden, unsere Anforderungen sind für diese Personen zu hoch. Insgesamt ist die Qualität der Ausbildungen in den letzten 15 Jahren sehr viel besser geworden, so dass wir kaum noch abenteuerliche oder absurde Registrierungsgesuche sehen. Dies ist nicht zuletzt auch den enormen Bemühungen der Verbände der Erfahrungsmedizin $\mathrm{zu}$ verdanken die über ihre Organisationen der $\mathrm{Ar}$ beitswelt grosse Arbeit leisten.

«Noch gibt es viel zu tun, aber die

Situation ist deutlich transparenter geworden.»

Welche gesetzlichen Regelungen gelten momentan bezüglich der Berufszulassung für nicht-ärztliche Therapeutinnen und Therapeuten?

Keine, sofern eidgenössische Gesetze gemeint sind. Kantonal gibt es Gesetze bezüglich Berufsausübungsbewilligung. Einige Kantone sind da sehr restriktiv bis ablehnend, andere sehr permissiv. Bei der Terminologie herrscht in den Kantonen zum Teil ein eindrückliches Durcheinander der Begrifflichkeiten. Das EMR hat die Gesetze aller Kantone in Hinblick auf die Erfahrungsmedizin analysiert und verglichen - da gibt es einige Widersprüche und auch richtige Fehler. Zudem kommen auf die Kantone neue Fragestellungen zu, für welche die Gesetze angepasst werden müssen, wie zum Beispiel bei den Themen Heilmittelabgabe, Mehrwertsteuer oder auch - die neueste Entwicklung - die Verordnung von Laboranalysen durch die Therapeuten. Meiner Meinung nach muss eindeutig klar sein, dass für solche Fragestellungen und Leistungen eine entsprechende Ausbildung vorhanden sein muss.

15 Jahre EMR, das bedeutet auch viele Daten und Erkenntnisse. Können Sie uns einige der interessantesten Zahlen aus dem EMR-Fundus nennen?

Mit 19300 Registrierungen gibt es fast 2000 EMRTherapeuten mehr als FMH-Ärzte im ambulanten Sektor. Jährlich melden sich etwa 1400 Therapeuten neu beim EMR an. Wir haben Kontakt zu rund 2700 Schulen und zu über 1000 Verbänden, die mit zum Teil divergierenden Interessen aktiv sind. Spitzenreiter bei den Top Ten der Methoden sind die Massagepraktiken. Zürich hat die meisten EMRTherapeuten, Appenzell Innerrhoden die wenigsten. Bei der Dichte pro 1000 Einwohner hat hingegen Appenzell Ausserrhoden die Nase vorn, gefolgt von Luzern und Basel-Stadt. Und, keine Zahl, aber eine wichtige Erkenntnis: Es fehlt (weltweit) immer noch die Antwort auf die Frage, was zur Erfahrungsmedizin gehört und was nicht.

\section{Das Wichtigste zum EMR}

Das ErfahrungsMedizinische Register EMR vergibt ein Qualitätslabel für erfahrungsmedizinische Therapeuten. Für die Erfahrungsmedizin, auch Komplementär- oder Alternativmedizin genannt, gibt es in der Schweiz keine einheitliche Regelung in Hinblick auf Ausbildung und Berufszulassung. Als unabhängige Institution definiert das EMR seit 15 Jahren einen Qualitätsstandard für diesen Bereich des Gesundheitswesens und leistet damit einen wichtigen Beitrag zum Patientenschutz. Mehr als 40 Schweizer Versicherer nutzen das EMR-Qualitätslabel als Voraussetzung, um erfahrungsmedizinische Leistungen im Rahmen einer privaten Zusatzversicherung zu erstatten. Derzeit sind über 19000 Therapeuten beim EMR registriert. Für die Öffentlichkeit bietet das EMR mit dem EMindex ein Internetverzeichnis an, mit dem Interessierte schnell und einfach einen Therapeuten mit EMR-Qualitätslabel finden können.

www.emr.ch • www.emindex.ch 
Die Erfahrungsmedizin gleicht einem Dschungel, der eine unglaubliche Vielfalt an Methoden und Therapeuten hervorbringt. Ist es möglich, auf diesem Gebiet den Überblick zu haben?

Der Dschungel hat sich in den letzten Jahren zu einer Naturwiese entwickelt. Noch gibt es viel zu tun, aber die Situation ist deutlich transparenter geworden. Nach 15 Jahren Erfahrung haben wir - ein Team von 50 Mitarbeitenden - inzwischen einen recht guten Überblick.

Können Sie uns einige besonders «exotische» Therapieverfahren nennen, die auf der Methodenliste des EMR eingetragen sind?

Exotisch ist natürlich ein relativer Begriff. Dem Wissenschaftler, der sich auf die Evidenz verlässt, werden viele Methoden auf der EMR-Methodenliste exotisch erscheinen. Exotisch im Sinn von «ausländisch» sind sicher die Methoden der asiatischen Medizin. Exotisch im Sinn von «aussergewöhnlich» sind für Schulmediziner wohl die meisten Methoden, die in der Erfahrungsmedizin angeboten

\section{«Dem EMR bleibe ich noch lange treu. Die Arbeit gefällt mir, wir sind ein tolles, motiviertes Team.»}

werden. Unter www.emindex.ch kann man sich übrigens zu allen Methoden, die das EMR vertritt, informieren - und auch gleich einen Therapeuten in der Nähe finden.

Unter Wissenschaftlern und Schulmedizinern gibt es immer noch grosse Vorbehalte gegenüber der Erfahrungsmedizin. Bekommen Sie diese Skepsis bis hin zur Ablehnung zu spüren?

Eigentlich nicht. Da ich fast 20 Jahre im Vorstand der SGIM aktiv war, ist recht gut bekannt, dass ich eine bekennende Schulmedizinerin bin. Bei meiner Arbeit geht es mir vor allem um den Patientenschutz. Aber es ist mir auch wichtig, dass nicht mit vorgefassten Meinungen «exotische» Angebote für kranke Menschen abgelehnt werden. Wir dürfen nicht vergessen, dass die Erfahrungsmedizin zu einem wesentlichen Teil von chronisch kranken und schulmedizinisch austherapierten Patienten genutzt wird. Seien wir also froh, dass es solche Angebote gibt, die diese Menschen auffangen und ihnen weitere Möglichkeiten zur Bewältigung ihrer gesundheitlichen Probleme anbieten. Viele Kollegen signalisieren mir im Gespräch, dass sie mit der Erfahrungsmedizin rein gar nichts anfangen können. Oft erlebe ich in der Diskussion dann aber, dass sich die anfänglich deutliche Ablehnung relativiert.

\section{Der EMR-Tag der Erfahrungsmedizin} In diesem Jahr feiert das EMR sein 15-jähriges Jubiläum mit dem Tag der Erfahrungsmedizin am 20. September 2014 im Congress Center Basel. Namhafte Referenten und ein breites Themenspektrum versprechen neue Blickwinkel auf die Erfahrungsmedizin: Mit dabei sind unter anderem Prof. em. Reinhard Saller, ehemaliger Inhaber des Lehrstuhls für Naturheilkunde an der Universität Zürich, der stellvertretende Chefarzt der Abteilung Psychosomatik am Universitätsspital Basel, Prof. Dr. Wolf Langewitz, der Buchautor und Volkswirtschaftler Prof. Mathias Binswanger sowie der Publizist und Philosoph Ludwig Hasler.

Programm und Anmeldung unter: www.congress-info.ch/emr2014

Für die Jubiläumstagung des EMR im September haben Sie trotz der Berührungsängste eines grossen Teils der «akademischen Elite» mit der Komplementärmedizin auch Referenten mit unbestrittenen wissenschaftlichen Meriten gewinnen können. Wie haben Sie das geschafft?

Das müssten Sie eigentlich die Referenten fragen, nicht mich. Eine Rolle spielt sicher, dass man sich kennt, sich vertraut. Aber wir wollen an der Tagung ganz bewusst nicht über die Erfahrungsmedizin im engeren Sinn reden, sondern wir kreisen mit allen Referenten um das Thema Gesellschaft und Gesundheit. Das ist für die Referentinnen und Referenten genauso interessant wie für die etwa 1000 Besucher, die wir erwarten. Vielleicht hat auch der grosse Erfolg unserer Tagung im Jahr 2009 zum 10-jährigen Bestehen des EMR dazu beigetragen, dass die Referenten (wieder) kommen.

Sie wurden dieses Jahr 60 Jahre alt - auch ein Moment, die kommenden Jahre zu planen. Wie sehen die nächsten 15 Jahre für das EMR aus? Wie lange bleiben Sie dem EMR noch treu?

Dem EMR bleibe ich noch lange treu. Die Arbeit gefällt mir, wir sind ein tolles, motiviertes Team. Aber ich werde mich in der kommenden Zeit etwas aus dem Tagesgeschäft zurückziehen und mich mehr der «Aussenpolitik» des EMR widmen. Dafür hat mir in den letzten Jahren etwas die Zeit gefehlt. Zudem haben wir neue Eisen im Feuer, unter den Stichworten «EMR Österreich» und «EMR Deutschland». Hier sind spannende Abklärungen im Gang. Seit ich 60 Jahre alt bin, geniesse ich die Arbeit viel mehr, denn ich «muss» nichts mehr, sondern ich wähle aus, was mich interessiert und fasziniert. Und da meine Fantasie nie still steht, ist das einfach toll. 